\title{
Validación de una Escala para Medir la Calidad Instruccional (CAIN) en Formadores
}

\author{
Validation of a Scale to Measure Instructional Quality in \\ Trainers
}

\author{
José Pablo Siqueiros Aguilera ${ }^{1}$ \\ Martha Olivia Peña Ramos ${ }^{1}$ \\ Jesús Tánori Quintana ${ }^{2}$ * \\ Jesús Francisco Laborín Álvarez ${ }^{1}$ \\ ${ }^{1}$ Centro de Investigación en Alimentación y Desarrollo A.C., México \\ ${ }^{2}$ Instituto Tecnológico de Sonora, México
}

\begin{abstract}
La educación de calidad es un objetivo primordial para políticas educativas, sobre todo en educación superior. No obstante, es difícil concebir un proceso de calidad sin una línea base; es decir, realizando una valoración del estado actual del objeto de estudio mediante instrumentos de medida que valoren el desempeño del docente desde diferentes perspectivas y considerando a los distintos actores del proceso educativo. Por lo tanto, el objetivo del presente trabajo es traducir, adaptar y validar una escala que mide la Calidad Instruccional (CI) desde la perspectiva del alumnado. Se trabajó con una muestra no probabilística de tipo intencional con 278 alumnos/as de tres escuelas normales y una subsede de la Universidad Pedagógica Nacional (UPN) campus Ciudad Obregón en el Estado de Sonora, México, en el ciclo 2017-2018. A través del análisis factorial confirmatorio se obtuvo la validez de constructo. Enseguida se efectuó un análisis de consistencia interna. El resultado obtenido sustenta la utilización del modelo para medir Calidad Instruccional en formadores sonorenses. Cabe mencionar que los valores de validez y confiabilidad hacen que el modelo de medida sea adecuado.
\end{abstract}

Palabras clave: Educación superior; Enseñanza; Evaluación del docente; instrumento de medida; Psicometría.

Quality education is a primary objective for educational policies, especially in higher education. However, it is difficult to conceive of a quality process without a baseline; that is, making an assessment of the current state of the object of study with the use of measuring instruments that assess teacher performance from different perspectives while considering the different actors in the educational process. Therefore, the aim of the present study is to translate, adapt and validate a scale which measures Instructional Quality from the perspective of the student. We worked with a non-probabilistic sample of 278 students from 3 normal schools and a sub-campus of UPN Ciudad Obregón campus in the state of Sonora, Mexico in the 2017-2018 cycle. The procedure for the analysis of the information begins with validation of confirmatory factor analysis, in addition to calculating reliability indexes. The results obtained support the use of the model to measure the construct of Instructional Quality in Sonoran trainers. It is worth mentioning that the values of validity and reliability make the measurement model sustainable.

Keywords: Higher education; Teaching; Teacher evaluation; Measuring instrument; Psychometrics.

*Contacto: jesus.tanori@itson.edu.mx

issn: 1989-0397

www.rinace.net/riee/

https://revistas.uam.es/riee
Recibido: 1 de mayo de 2019

$1^{\text {a }}$ Evaluación: 27 de julio de 2019

$2^{\text {a }}$ Evaluación: 8 de septiembre de 2019

Aceptado: $\quad 22$ de septiembre de 2019 


\section{Introducción}

Las prácticas que el profesorado desarrolla en el aula (como el manejo de clase, la motivación del alumnado, el tiempo y nivel de instrucción, por mencionar algunas), constituyen un elemento que determina la instrucción efectiva. En el caso del formador de docentes el reto radica en la capacidad de integrar diferentes tipos de dominios: la formación pedagógica, la formación disciplinar, el conocimiento didáctico de lo que se enseña, así como la apreciación y conocimiento del contexto en el que se enseña (Slavin, 1994; Vaillant, 2004; Wagner, Göllner, Helmke, Trautwein y Lüdtke, 2013).

En México la instrucción es evaluada en funciones del desempeño donde se consideran cuatro etapas que involucran evaluaciones por superiores, expedientes de evidencias, exámenes de conocimientos y planeaciones de las clases. De la evaluación del 2015 a la del 2016 se observaron mejoras en todos los niveles, en particular las planeaciones. Esto puede tener relación con el tipo de participación; mientras que en la evaluación obligatoria (la de 2015) intervinieron 134,285 docentes, en la voluntaria 23,911, todo esto en educación básica y media superior (INEE, 2018).

En un estudio internacional sobre la configuración de la enseñanza y el aprendizaje (TALIS, siglas del inglés Teaching and Learning International Survey) los docentes mexicanos reportan un alto uso de prácticas de enseñanza activa. Cabe mencionar que en dicho estudio México tiene el menor índice de formación docente dentro de los países pertenecientes a la Organización para la Cooperación y Desarrollo Económico (OCDE), el cual está relacionado con los resultados del alumnado y a la autoeficacia del docente (Backhoff, Vázquez-Lira, Baroja, Guevara y Morán, 2017). Sin embargo, en otro estudio internacional (PISA, por sus siglas en inglés, Programme for International Student Assessment), la autoeficacia reportada es alta para estudiantes mexicanos, pero los resultados de desempeño son bajos (Burns y Darling-Hammond, 2014). Por lo tanto, se considera determinante contar con una alternativa de evaluación distinta al autoinforme con el fin de que esta incluya otros elementos importantes a la mejora de las prácticas educativas, como lo es la percepción del alumnado.

Con base en lo anterior, la necesidad de involucrarse en actividades de formación profesional es indudable si se persigue una educación de calidad. Para el profesorado mexicano de escuelas normales (EN), no es una excepción si tomamos en consideración que en el ciclo 2015-2016 trabajaron 12,097 docentes formadores en escuelas públicas y 3,505 en escuelas normales privadas, de los cuales el $57,4 \%$ y $59,7 \%$, respectivamente terminaron la licenciatura. Es importante mencionar que el 40,6\% de las y los docentes del sector público contaban con posgrado, comparado con un 39,5\% del sector privado, el resto se ubica en la población de docentes con licenciatura incompleta, el $2 \%$ para el sector público y el 0.8\% para el privado (Medrano, Ángeles y Morales, 2017).

Ante este contexto, analizar las prácticas docentes desde la perspectiva del alumnado cobra importância, ya que permite identificar áreas de mejora para el desarrollo profesional del docente. Una de las variables que predicen la participación en actividades de desarrollo profesional y el sentido de preparación de los docentes es la calidad de la instrucción (Blömeke, Olsen y Suhl, 2016). 


\section{Calidad de la instrucción: referentes teóricos}

La teoría de la instrucción se integra por la identificación de aquellos métodos que provean las mejores condiciones para que se logren los objetivos de aprendizaje. Para lograr dichos objetivos se deben seguir principios universales, los cuales pueden ser aplicables en cualquier situación de enseñanza, ya que proveen un resumen de las acciones más importantes para la alta calidad de la instrucción (Merrill, 2002; Merrill, Barclay y van Schaak, 2008; Reigeluth, 2016).

Los principios de la instrucción se alinean con los principios de enseñanza efectiva de Brophy (2001), quien propuso una síntesis de principios (ver cuadro 1) que van desde aspectos de curriculum, instrucción y evaluación a elementos dentro del aula como prácticas de organización y gestión.

Cuadro 1. Principios de enseñanza efectiva de Brophy (2001)

\begin{tabular}{ll}
\hline \multicolumn{1}{c}{ PRINCIPIO } & \multicolumn{1}{c}{ CATEGORÍA } \\
\hline Un clima de apoyo en el aula & Manejo de clase \\
$\begin{array}{l}\text { Enseñanza estratégica } \\
\text { Aprendizaje cooperativo }\end{array}$ & \\
\hline Oportunidad de aprender & Participación del alumno \\
Discurso reflexivo & \\
Actividades de práctica y aplicación & \\
Andamios de compromiso de tareas de los estudiantes & \\
Expectativas de logro & Estructura de clase \\
\hline Alineación curricular & \\
Establecimiento de orientaciones de aprendizaje & \\
Contenido coherente & \\
Evaluación orientada a los objetivos &
\end{tabular}

Fuente: Elaboración propia.

Dentro de las variables relacionadas a la calidad de la instrucción (CI) podemos encontrar aquellas referidas al docente como autoeficacia (Holzberger, Philipp y Kunter, 2013), o los resultados de los alumnos, como las pruebas internacionales PISA o TIMMS (Klette, Blikstad-balas y Roe, 2017; Nilsen y Gustafsson, 2016). También encontramos aquellas que se determinan por el contexto o las características de la clase. En particular la percepción de rápida instrucción y baja carga de trabajo pueden predecir el alto absentismo escolar (Sälzer, Trautwein, Lüdtke y Stamm, 2012).

En el modelo QAIT (por sus siglas en inglés Quality of instruction, Appropriate levels of instruction, Incentives and Time) se integran elementos, tanto contextuales como aquellos referidos al docente. La calidad de la instrucción, el nivel apropiado de la misma, así como los incentivos o formas de motivar y el tiempo para la instrucción, son dimensiones que definen la instrucción efectiva según Slavin (1994).

En la revisión sobre el constructo CI, se identificó que en la mayoría fueron realizados en idioma inglés y alemán; así como en un context europeo (Blömeke, Olsen y Suhl, 2016; Holzberger, Philipp y Kunter, 2013) y estadounidense (Junker et al., 2006). Para el caso de América Latina -y en idioma español- la búsqueda resultó escassa. Se encontró un estudio de Sánchez-Rosas y Esquivel (2016), sin embargo está redactado en idioma inglés. Lo que significa un problema para la evaluación del desempeño del docente desde una perspectiva diferente de la autoevaluación o autoreporte. El idioma representa una desventaja, tanto como el lenguaje. 
Para medir la CI podemos tomar la perspectiva del alumnado, lo cual nos permite mayor número de participantes y facilita la aplicación del instrumento. Se ha encontrado a nivel básico, medio y medio superior, que los estudiantes pueden proporcionar evaluaciones diferenciadas de las dimensiones de la CI, así como la estabilidad del constructo a través de clases y materias (Praetorius, Pauli, Reusser, Rakoczy y Klieme, 2014; Wagner et al., 2013), pero no para niveles universitarios o superiores dentro de los cuales están las EN.

La evaluación docente universitaria, a través del alumnado, es uno de los mejores métodos, ya que el alumno es el observador permanente de la actuación docente. Además, desde la perspectiva del alumnado se puede resaltar la calidad de las interacciones dentro del aula, aunado a la efectividad del método en la reducción de costos (Downer, Stuhlman, Schweig, Martínez y Ruzek, 2014; Tejedor, 2012).

Uno de los propósitos de la Ley General del Servicio Profesional Docente (LGSPD), expedida en 2013, fue mejorar la práctica profesional mediante la evaluación en las escuelas y para tal empresa el Instituto Nacional para la Evaluación de la Educación (INEE, 2018) destacaba la evaluación interna y externa como componentes para el desarrollo profesional docente en educación básica, pues emite directrices asociadas a problemas, dentro de los cuales incide la insuficiente articulación entre la formación inicial y continua y la falta de acciones para la formación de docentes.

Así como la evaluación es un factor que sirve de guía para el docente en relación al aprendizaje del alumno, también lo es para orientar el proceso de enseñanza del docente, pero esta ha de ser frecuente, cercana al alumnado y con retroalimentación rápida para que tenga impacto en el aula, de ahí la importancia de instrumentos que den cuenta de lo que ocurre dentro del aula (Murillo, Martínez-Garrido y Hernández, 2011).

Con base en la necesidad de contar con instrumentos en idioma español sobre procesos de instrucción a nivel superior o universitario, el propósito del presente estudio es: traducir, adaptar y validar una escala que mide la CI desde la perspectiva del alumnado para una población universitaria del Noroeste de México, en particular determinar la validez y confiabilidad de la escala de calidad instruccional de Wagner et al. (2013), así como de sus dimensiones: motivación, comprensión, participación del estudiante, estructura de la clase y manejo de la clase.

\section{Diseño metodológico}

Se realizó un estudio de naturaleza cuantitativa, alcance descriptivo y tipo no experimental para determinar la validez y confiabilidad de un modelo de medida (Johnson y Christensen, 2017).

\subsection{Participantes}

En el estado de Sonora existen ocho escuelas normales (EN) ubicadas en cuatro municípios: Hermosillo, Cajeme, Navojoa y Etchojoa, además, se cuenta con nueve sedes de la Universidad Pedagógica Nacional (UPN) en los municípios de Agua Prieta, Caborca, Guaymas, Hermosillo, Navojoa, Nogales, Huatabampo, Cajeme y San Luis Río Colorado, que albergan una población estudiantil de formación inicial (licenciaturas) de 4.166 alumnos(as) en once programas (CRESON, 2018).

Se trabajó con una muestra no probabilística de tipo intencional de 278 alumnos, de los cuales el $82 \%$ eran mujeres y el $18 \%$ hombres, proporción cercana a la nacional que en el 
ciclo 2018-2019 tuvo un 74,2\% mujeres y 25,8\% hombres (SIBEN, 2018). La edad promedio fue de 19 años en un rango de 18 a 41. La mayoría de los respondientes se ubicaron en el municipio de Hermosillo 176 (63\%) y el resto en Cajeme. Participaron cuatro escuelas, tres normales (educación preescolar, primaria, especial y educación física) y una UPN (de licenciatura cuatrimestral en educación preescolar y primaria), las cuales ofertan seis de las 11 licenciaturas. Para el levantamiento de datos se contó con un formato de consentimiento informado individual y la solicitud por escrito a los directivos de las instituciones participantes.

\subsection{Instrumento. El constructo de calidad instruccional}

Para medir la calidad instruccional se utilizaron las cinco dimensiones de la escala de 16 reactivos de Wagner y otros (2013). Dicho modelo tiene un soporte teórico adecuado (CFI=0,974; RMSEA=0,027) y las cinco dimensiones son: motivación, comprensión, participación del estudiante, estructura de la clase y manejo de la clase, con escala de respuesta tipo Likert de $\mathrm{O}=$ Totalmente en desacuerdo a $4=$ Totalmente de acuerdo. Cabe mencionar que en la descripción de la escala se recomienda un análisis por dimensiones para futuras investigaciones.

En el cuadro 2, podemos observar la descripción de la escala original de cinco factores, de acuerdo con la definición de cada dimensión y los reactivos que la componen.

Cuadro 2. Dimensiones y reactivos de la escala de calidad instruccional

\begin{tabular}{|c|c|c|}
\hline DIMENSIÓN & DEFINICIÓN & REACTIVOS \\
\hline Motivación & $\begin{array}{l}\text { Capacidad de los docentes para } \\
\text { promover la motivación de los } \\
\text { estudiantes en los temas } \\
\text { cubiertos en las lecciones. }\end{array}$ & $\begin{array}{l}\text { A veces mi profesor(a) realmente me } \\
\text { entusiasma con los temas de clase. } \\
\text { Mi maestro(a) puede convertir un tema poco } \\
\text { inspirador en uno interesante. }\end{array}$ \\
\hline Comprensión & $\begin{array}{l}\text { Comprensión del } \\
\text { comportamiento de los docentes, } \\
\text { que se refiere al énfasis y los } \\
\text { enfoques que usa para ayudar a } \\
\text { los estudiantes a entender. }\end{array}$ & $\begin{array}{l}\text { Las tareas de una clase son claras y } \\
\text { comprensible para mí. } \\
\text { Cuando mi maestro(a) explica algo, él o ella } \\
\text { usa claros ejemplos. } \\
\text { Mi maestro(a) habla articulada y claramente. } \\
\text { Mi maestro(a) explica las cosas en una forma } \\
\text { que sea comprensible para mí. }\end{array}$ \\
\hline $\begin{array}{l}\text { Participación } \\
\text { del estudiante }\end{array}$ & $\begin{array}{l}\text { Percepción de los estudiantes } \\
\text { sobre el interés de los maestros } \\
\text { en sus ideas y opiniones. }\end{array}$ & $\begin{array}{l}\text { Mi maestro(a) atiende a nuestras sugerencias. } \\
\text { Mi maestro(a) nos anima a expresar nuestras } \\
\text { opiniones personales. } \\
\text { Si alguien tiene una buena idea, mi } \\
\text { maestro(a) responde a eso. } \\
\text { Mi maestro(a) me da la oportunidad de } \\
\text { expresar mi opinión. } \\
\text { A mi maestro(a) le interesa lo que digo. }\end{array}$ \\
\hline $\begin{array}{l}\text { Estructura de } \\
\text { la clase }\end{array}$ & $\begin{array}{l}\text { Grado en que los objetivos de la } \\
\text { lección fueron claros para los } \\
\text { estudiantes y el grado en que la } \\
\text { secuencia de la lección fue } \\
\text { estructurada para construir } \\
\text { comprensión y mantener un } \\
\text { sentido de propósito. }\end{array}$ & $\begin{array}{l}\text { Al comienzo de una clase, mi maestro(a) } \\
\text { describe lo que se van a cubrir. } \\
\text { Al final de una lección, mi maestro(a) resume } \\
\text { los puntos más importantes. } \\
\text { Mi maestro(a) proporciona información sobre } \\
\text { los aspectos más importantes de la clase. }\end{array}$ \\
\hline $\begin{array}{l}\text { Manejo de la } \\
\text { clase }\end{array}$ & $\begin{array}{l}\text { Grado en que el docente nota } \\
\text { interrupciones o falta de atención } \\
\text { del estudiante. }\end{array}$ & $\begin{array}{l}\text { Mi maestro(a) logra que las y los estudiantes } \\
\text { pongan atención a la clase. } \\
\text { Mi maestro(a) se da cuenta todo lo que } \\
\text { sucede en clase. }\end{array}$ \\
\hline
\end{tabular}

Fuente: Elaboración propia. 


\subsection{Procedimiento}

Se realizó un estudio piloto para la validación de la escala, el cual inició con el proceso de traducción para posteriormente someter los reactivos a una re-traducción (Brislin, 1970; Guo, 2017).

La versión final en español se consideró equivalente a la versión inglesa, con el apoyo de dos jueces bilingües y con dominio de lenguaje técnico en el área de psicología y educación. Se aplicó a alumnos de escuelas normales (de las licenciaturas en preescolar, primaria y educación física) y UPN (del programa o licenciatura en educación preescolar y primaria, el cual es cuatrimestral), previa gestión de oficios de presentación y consentimiento dirigidos al personal directivo de cada escuela, además de informar sobre la confidencialidad y manejo adecuado de la información a cada participante.

Estos datos se manejaron con el Paquete Estadístico para las Ciencias Sociales SPSS 22 y la extensión Amos 22 para el realizar el Análisis Factorial Confirmatorio. Finalmente se calcularon los índices de confiabilidad, con el objetivo de complementar al modelo de medida y de esta forma establecer la certeza de variabilidad de las respuestas de los participantes.

\section{Resultados}

\subsection{Análisis Factorial Confirmatorio}

La escala original de CI está basada en cinco factores o dimensiones: motivación, comprensión, participación del estudiante, estructura de la clase y manejo de la clase. Se realizó un Análisis Factorial Confirmatorio (AFC) para determinar las propiedades psicométricas del modelo, del cual se obtuvieron valores fuera de lo esperado, es decir, el modelo es inadecuado. Asimismo, para el modelo de cuatro factores, los resultados no fueron los esperados y se eliminaron siete reactivos y se ajustó a un modelo de tres factores (como se observa en el cuadro 3). Los valores de referencia se tomaron de la validación de Valdés, Carlos, Wendlandt y Ramírez (2016).

Cuadro 3. Indicadores de ajuste del modelo estructural

\begin{tabular}{|c|c|c|c|c|}
\hline INDICADOR & $\begin{array}{c}\text { VALOR } \\
\text { ADECUADO }\end{array}$ & $\begin{array}{c}\text { CINCO } \\
\text { FACTORES }\end{array}$ & $\begin{array}{l}\text { CUATRO } \\
\text { FACTORES }\end{array}$ & $\begin{array}{c}\text { TRES } \\
\text { FACTORES }\end{array}$ \\
\hline Razón x2/gl & $\geq 2.00$ & 2,47 & 1,95 & 1,64 \\
\hline $\begin{array}{l}\text { Indice de bondad de ajuste Joreskog } \\
\text { (GFI) }\end{array}$ & $\geq 0,90$ & 0,62 & 0,91 & 0,96 \\
\hline $\begin{array}{l}\text { Indice de ajuste normado de Bentler- } \\
\text { Bonett (NFI) }\end{array}$ & $\geq 0,90$ & 0,94 & 0,55 & 0,98 \\
\hline $\begin{array}{l}\text { Indice comparativo de ajuste de } \\
\text { Bentler (CFI) }\end{array}$ & $\geq 0,93$ & 0,75 & 0,70 & 0,99 \\
\hline $\begin{array}{l}\text { Error cuadrático medio de } \\
\text { aproximación (RMSEA) }\end{array}$ & $\leq \mathrm{0}, 05$ (ideal) & 0,07 & 0,59 & 0,04 \\
\hline
\end{tabular}

Fuente: Elaboración propia.

En la imagen 1 se pude observar el modelo estructural de la escala CI ajustado a tres factores que mantienen una validez de constructo en lo que refiere a enseñanza efectiva, que se compone de aspectos como curriculum, instrucción y evaluación, así como de elementos del aula, prácticas de organización y gestión (Brophy, 2001). 
El factor 1 o "F1" corresponde a la dimensión de participación del estudiante y se compone de cuatro reactivos (7,8, 9 y 10), el segundo factor o "F2" corresponde a la dimensión de estructura de clase y se compone de tres reactivos (12, 13 y 14). Por último, el tercer factor o "F3" se compone de dos reactivos (15 y 16) de la dimensión de manejo de clase, estos dos últimos factores se componen de los mismos elementos que la escala original (ver figura 3). Cabe mencionar que para la determinación de los factores los criterios suelen ser variados; sin embargo, valores de ajuste para el modelo de tres factores resultaron satisfactorios de acuerdo a lo expuesto por Coubergs, Struyen, Vanthournout y Engels (2017), por lo que se puede asumir que el modelo es válido o sustentable.

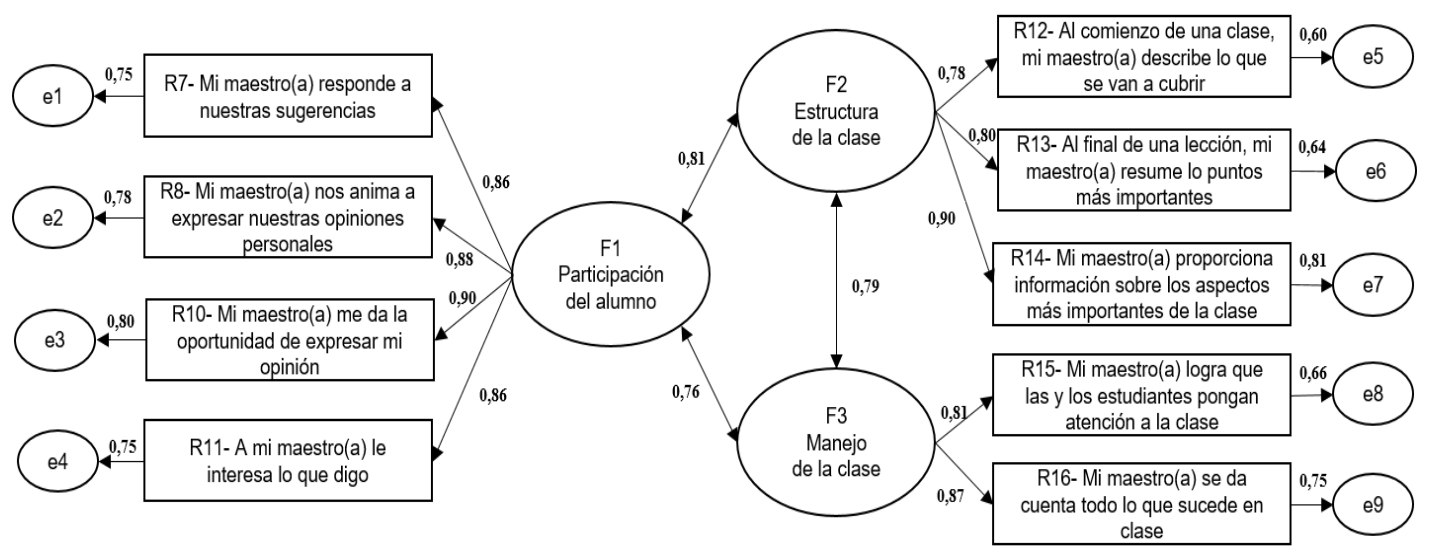

Figura 1. Modelo estructural de la escala CI Fuente: Elaboración propia.

\subsection{Confiabilidad}

La confiabilidad se estimó con el índice del alfa de Cronbach, el cual se hizo por cada factor tal como se observa en el cuadro 4. Siendo todos por dimension y el total de la escala, los valores del indice de consistencia interna adecuados, ya que oscilan ente 0,82 y 0,93. Por lo tanto podemos decir que es consistente al momento de aplicarla (Oviedo y Campo-Arias, 2005).

Cuadro 4. Confiabilidad global y por factor

\begin{tabular}{lc}
\hline & ALFA DE CRONBACH \\
\hline Factor 1: Participación del alumno & 0,92 \\
Factor 2: Estructura de clase & 0,86 \\
Factor 3: Manejo de clase & 0,82 \\
Global CI & 0,93 \\
\hline
\end{tabular}

Fuente: Elaboración propia.

\section{Discusión y conclusiones}

Por lo anterior, se puede asumir que el instrumento analizado presenta propiedades psicométricas que lo hacen pertinente y confiable para medir tres factores del constructo de calidad instruccional. Los resultados obtenidos sustentan la utilización del modelo para medir el constructo en población académica específica, en este caso en el estado de Sonora, que se ubica al noroeste de México (ver anexo I). Cabe mencionar, además, que los valores de validez y confiabilidad hacen un modelo de medida sustentable, sin 
embargo, el modelo ajustado de tres dimensiones hacen referencia al hacer y no al Ser como era el caso de las dimensiones motivación y comprensión que estaban compuestas por los reactivos eliminados, lo que representa una oportunidad para evaluar dicho constructo con otros asociados como lo reportaron Holzberger, Philipp y Kunter (2013).

Por otro lado, la eliminación de los reactivos (y con ello la desaparición de dos factores) pueden ser debido a la cultura en la cual, han sido elaborados, aplicados y validados (Furr y Bacharach, 2014; Reyes-Lagunes, 1993). Por eso, en este trabajo se tuvo como objetivo traducir, adaptar y evaluar las propiedades psicométricas de un modelo de medición de calidad instruccional.

Se concluye entonces, que las propiedades que presenta la escala la hacen pertinente en la investigación y la gestión académica, ya que al contar con un instrumento que mida la calidad con la que el docente instruye o enseña, permitirá a las y los directivos tener más certeza en la toma de decisiones de capacitación y de procesos de mejora de la eficacia escolar en las instituciones formadoras. Lo anterior constituye una aportación al campo educativo de la investigación sobre la formación docente como objeto de estudio; ya que la indagación de dicha figura en Latinoamérica es una línea de generación de conocimiento en construcción. En la actualidad son pocos los trabajos publicados en idioma español en comparación con las disponibles en idioma inglés y puede tener relación con la diversidad de expresiones lingüísticas para nombrar o identificar al formador de formadores (González-Vallejos, 2018).

En sentido práctico, la presente validación aporta un instrumento breve en lo que a número de reactivos refiere, además de una validez y fiabilidad adecuadas para medir la calidad instruccional a nivel superior. Asimismo cabe destacar el aporte metodológico del presente trabajo al dotar de una herramienta al estudio de la calidad instruccional. De igual forma se presentan un par de limitaciones: la primera de ellas consiste en que es la misma población, ya que el número de alumnos que cursan programas dentro de las escuelas que preparan formadores es bajo; lo que restringe el proceso de adaptar y validar las pruebas en culturas distintas (Góngora, 2000; Hair, Black, Barbin y Anderson, 2010); la segunda corresponde a la prueba original, la cual tenía en dos dimensiones un grupo restringido de reactivos (Morales, 2011); por lo que se sugiere seguir abonando a la escala ítems que representen el modelo original (como lo recomiendan los creadores del instrumento de medida).

\section{Referencias}

Backhoff, E., Vázquez-Lira, R., Baroja, J. L., Guevara, G. P. y Morán, Y. (2017). México en el proyecto TALIS-PISA: Un estudio exploratorio. Importancia de las escuelas, directores, docentes y estudiantes en el aprendizaje de las matemáticas. Ciudad de México: INEE.

Blömeke, S., Olsen, R. V. y Suhl, U. (2016). Relation of student achievement to the quality of their teachers and instructional quality. En T. Nilsen y J. Gustafsson (Eds.), Teacher Quality, Instructional Quality and Student Outcomes (pp. 21-50). Ginebra: Springer.

Brislin, R. W. (1970). Back-Translation for Cross-Cultural Research. Journal of Cross-Cultural Psychology, 1(3), 185-216. https://doi.org/10.1177/135910457000100301

Brophy, J. (2001). Generic aspects of effective teaching. Tomorrow's Teachers, 1, 3-45. https://doi.org/10.1016/So167-8922(00)80004-8 
Burns, D. y Darling-Hammond, L. (2014). Teaching around the world: What can TALIS tell us. Stanford Center for Opportunity Policy in Education. Recuperado de https://edpolicy.stanford.edu/sites/default/files/publications/teaching-around-worldwhat-can-talis-tell-us_3.pdf

Coubergs, C., Struyven, K., Vanthournout, G. y Engels, N. (2017). Measuring teachers' perceptions about differentiated instruction: The DI-Quest instrument and model. Studies in Educational Evaluation, 53, 41-54. https://doi.org/10.1016/j.stueduc.2017.02.004

CRESON. (2018). Estadísticas y control escolar. Base de datos. Recuperado de http://www.creson.edu.mx/

Downer, J., Stuhlman, M., Schweig, J., Martínez, J. y Ruzek, E. (2015). Measuring effective teacher-student interactions from a student perspective: A multi-level analysis. The Journal of Early Adolescence, 35(5-6), 722-758.

https://doi.org/10.1177/0272431614564059

Furr, M. y Bacharach, V. (2014). Psychometrics. An introduction. Nueva York, NY: Sage.

Góngora, E. (2000). El enfrentamiento a los problemas y el papel del control: una visión etnopsicológica en un ecosistema con tradición. Tesis Doctoral. Universidad Autónoma de México, Ciudad de México, México.

González-Vallejos, M. (2018). El estudio del formador latinoamericano: un campo de investigación "en construcción”. Magis, Revista Internacional de Investigación en Educación, 1O(21), 35-54. https://doi.org/10.11144/Javeriana.m10-21.eflc

Guo, T. (2017). On Foreign Language Creation and Rootless Back Translation-A Case Study of Snow Flower and the Secret Fan. Journal of Literature and Art Studies, 7(10), 1354-1364. https://doi.org/10.17265/2159-5836/2017.10.017

Hair, J. F., Black, W. C., Babin, B. J. y Anderson, R. E. (2010). Multivariate data analysis. Trenton, NJ: Pearson Prentice Hall.

Holzberger, D., Philipp, A. y Kunter, M. (2013). How teachers' self-efficacy is related to instructional quality: A longitudinal analysis. Journal of Educational Psychology, 105(3), 774-786. https://doi.org/10.1037/a0032198

INEE. (2018). La educación obligatoria en México. Informe 2018. Ciudad de México: INEE.

Johnson, B. y Christensen, L. (2017). Educational research: quantitative, qualitative and mixed approaches. Thousand Oaks, CA: Sage.

Junker, B. W., Weisberg, Y., Matsumura, L., Crosson, A., Wolf, M., Levison, A. y Resnick, L. (2006). Overview of the instructional quality assessment. Los Angeles, CA: Regents of the University of California.

Klette, K., Blikstad-balas, M. y Roe, A. (2017). Linking Instruction and Student Achievement Research design for a new generation of classroom studies $A$ koble undervisning med elevprestasjoner - Forskningsdesign for en ny generasjon klasseromsstudier, 11(3), 1-19.

Medrano, V., Ángeles, E. y Morales, M. (2017). La educación normal en México. Elementos para su análisis. Ciudad de México: INEE.

Merrill, M. D. (2002). First principles of instruction. Educational Technology Research and Development, 50(3), 43-59. https://doi.org/10.1007/BF02505024

Merrill, M. D., Barclay, M. y van Schaak, A. (2008). Prescriptive principles for instructional design. Handbook of research on educational communications and technology, Lawrence Erlbaum Associates. Nueva York, NY: Routledge, Taylor and Francis Group. 
Morales, P. (2011). El Análisis Factorial en la construcción e interpretación de tests, escalas y cuestionarios. Madrid: Universidad Pontificia Comillas.

Murillo, F. J., Martínez-Garrido, C. y Hernández, R. (2011). Decálogo para una enseñanza eficaz. REICE. Revista Iberoamericana sobre Calidad, Eficacia y Cambio en Educación, 9(1), 7-27.

Nilsen, T. y Gustafsson, J. (2016). Teacher quality, instructional quality and student outcomes. Londres: Springer. https://doi.org/10.1007/978-3-319-41252-8

Oviedo, C. y Campo-Arias, A. (1995). Aproximación al uso del coeficiente alfa de Cronbach. Revista Colombiana de Psiquiatría, 34(4), 572-580.

Praetorius, A. K., Pauli, C., Reusser, K., Rakoczy, K. y Klieme, E. (2014). One lesson is all you need? Stability of instructional quality across lessons. Learning and Instruction, 31, 2-12. https://doi.org/10.1016/j.learninstruc.2013.12.002

Reigeluth, C. (2016). Instructional theory and technology for the new paradigm of education. Revista de Educación a Distancia, 50, 1-18. https://doi.org/10.4324/9781315760933

Reyes-Lagunes, I. (1993). Las redes semánticas naturales, su conceptualización y su uso en la construcción de instrumentos. Revista de Psicología Social y Personalidad, 9, 83-99.

Sälzer, C., Trautwein, U., Lüdtke, O. y Stamm, M. (2012). Predicting adolescent truancy: The importance of distinguishing between different aspects of instructional quality. Learning and Instruction, 22(5), 311-319. https://doi.org/10.1016/j.learninstruc.2011.12.001

Sánchez-Rosas, J. y Esquivel, S. (2016). Instructional Teaching Quality, Task Value, SelfEfficacy, and Boredom: A Model of Attention in Class. Revista de Psicología, 25(2), 1-20. https://doi.org/10.5354/0719-0581.2017.44966

SIBEN. (2018). Matrícula ciclo escolar 2017-2018. Recuperado de http://www.siben.sep.gob.mx/pages/estadisticas_recientes

Slavin, R. (1994). Quality, appropriateness, incentive, and time: A model of instructional effectiveness. International Journal of Educational Research, 21(2), 141-157. https://doi.org/10.1016/0883-0355(94)90029-9

Tejedor, F. J. (2012). Evaluación del desempeño docente. Revista Iberoamericana de Evaluación Educativa, 5(1e), 319-327.

Vaillant, D. (2004). Construcción de la profesión docente en América Latina: tendencias, temas y debates. Santiago de Chile: PREAL.

Valdés, A., Carlos, A., Wendlandt, T. y Ramírez, M. (2016). Propiedades psicométricas de una escala para medir el manejo de la vergüenza en adolescentes (MOSS-SAST). Acta Colombiana de Psicología, 19(1), 13-34. https://doi.org/10.14718/ACP.2016.19.1.2

Wagner, W., Göllner, R., Helmke, A., Trautwein, U. y Lüdtke, O. (2013). Construct validity of student perceptions of instructional quality is high, but not perfect: Dimensionality and generalizability of domain-independent assessments. Learning and Instruction, 28, 1-11. https://doi.org/10.1016/j.learninstruc.2013.03.003 


\section{Anexo I}

\section{Instrumento CAIN-VA}

Instrucciones: A continuación, se le pide que emita su opinión a cada enunciado que se presenta. Debe mostrar su acuerdo en una escala de 0 a 4, de tal manera que 0 indicará total desacuerdo y 4 total acuerdo. No hay respuestas buenas o malas, por lo que se le pide sinceridad en las respuestas ya que éstas serán anónimas y confidenciales. Gracias por su participación.

Antes de iniciar se debe identificar una clase a evaluar (ej. Adecuación curricular), con base en esa clase se contestará la siguiente información.

Clase a evaluar:

\begin{tabular}{|c|c|c|c|c|c|}
\hline & $\mathrm{O}$ & 1 & 2 & 3 & 4 \\
\hline Mi maestro(a) responde a nuestras sugerencias & & & & & \\
\hline Mi maestro(a) nos anima a expresar nuestras opiniones personales & & & & & \\
\hline Mi maestro(a) me da la oportunidad de expresar mi opinión & & & & & \\
\hline A mi maestro(a) le interesa lo que digo & & & & & \\
\hline Al comienzo de una clase, mi maestro(a) describe lo que se van a cubrir & & & & & \\
\hline Al final de una lección, mi maestro(a) resume lo puntos más importantes & & & & & \\
\hline $\begin{array}{l}\text { Mi maestro(a) proporciona información sobre los aspectos más } \\
\text { importantes de la clase }\end{array}$ & & & & & \\
\hline Mi maestro(a) logra que las y los estudiantes pongan atención a la clase & & & & & \\
\hline Mi maestro(a) se da cuenta todo lo que sucede en clase & & & & & \\
\hline
\end{tabular}

\section{Breve Cv de los autores}

\section{José Pablo Siqueiros Aguilera}

Estudiante de Doctorado en Desarrollo Regional (candidato a Doctor) en el Centro de Investigación en Alimentación y Desarrollo (CIAD) y Maestro en Desarrollo Regional por el mismo Centro. Licenciado en Psicología por la Universidad de Sonora. Experiencia en docencia a nivel media superior. Así como en investigación y docencia en el área de formación de formadores. ORCID ID: https://orcid.org/0000-0003-21109999. Email: jpsa_mexico@hotmail.com

\section{Martha Olivia Peña Ramos}

Doctora en Educación, Maestría en Desarrollo Regional y Licenciada en Psicología, com especialidad, Educativa. Investigadora titular del Departamento del Desarrollo Humano y Bienestar Social de la Coordinación de Desarrollo Regional del CIAD, AC. Miembro fundador y parte del consejo técnico de la Red Temática Conacyt de Investigación en Educación Rural. Líneas de investigación: Educación rural y ambiental, etnopsicología, psicología transcultural, crianza y desarrollo infantil, apacitación docente y evaluación de políticas públicas en educación. ORCID ID: https://orcid.org/0000-0002-5781-8781. Email: mpena@ciad.mx 


\section{Jesús Tánori Quintana}

Doctor en Ciencias Sociales, con énfasis en Psicología Social, por la Universidad Autónoma de Sinaloa. Profesor Investigador Auxiliar en el Departamento de Educación del Instituto Tecnológico de Sonora. Áreas de interés: Factores psicosociales y de perosnalidad del comportamientos de escolares, calidad de vida y bienestar subjetivo, en población de infantil y adoslcente, evaluación docente y psicometría. Miembro del Sistema Nacional de Investigadores, Nivel 1 (SNI-1), CONACYT-México. ORCID ID: https://orcid.org/0000-0002-6485-2267. Email: jesus.tanori@itson.edu.mx

\section{Jesús Francisco Laborin Álvarez}

Doctor en Ciencias Sociales por la Universidad Autónoma de Sinaloa. Maestro en Psicología Social por la Universidad Nacional Autónoma de México. Licenciado en Psicología por la Universidad de Sonora. Investigador titular en la Coordinación de Desarrollo Regional. Centro de Investigación en Alimentación y Desarrollo, A.C. Áreas de interés: Factores psicosociales del desarrollo humano, calidad de vida y bienestar subjetivo, acompañamiento docente y etnopsicometría. Miembro del Sistema Nacional de Investigadores (SNI), CONACYT-México. ORCID ID: https://orcid.org/oooo0003-0747-6426. Email: laborin@ciad.mx 